\title{
Shape effects in optical properties of composite dust particles
}

\author{
Robert S. Botet ${ }^{1}$ and Rakesh K. Rai ${ }^{1,2}$ \\ ${ }^{1}$ Laboratoire de Physique des Solides-Bât.510 — CNRS UMR8502, Université Paris-Sud, 91405 Orsay, France \\ ${ }^{2}$ D. D. U. Gorakhpur University, 273009 Gorakhpur, India
}

(Received October 31, 2012; Revised March 15, 2013; Accepted March 20, 2013; Online published October 24, 2013)

\begin{abstract}
We present optical features characteristic of the shape of composite dust particles, such as grain-aggregates formed under cosmic or atmospheric conditions. The discussion develops along two examples of realistic models for the formation of the composite particles, and constant refractive indices. That way, particular features exhibited in the optical cross section behaviors result from the particle structure only. The role of the ratio between optical particle cross section and the corresponding cross section of the coated sphere of same composition and volume is highlighted. Limited wavelength ranges are defined where the coated sphere model can be used to obtain the effective radius or the volume composition of the particle. Other wavelength domains are very dependent on the particle formation mechanisms, then giving constraints on the possible formation processes. Therefore, such an approach is able to provide inverse methods to obtain the actual values of physical parameters from definite features of optical quantities.
\end{abstract}

Key words: Aggregate aerosol, composite particles, cosmic dust, extinction, fractal aggregates.

\section{Statement of the Problem}

In the interstellar medium, strong optical extinction of dust particles in the ultraviolet region is interpreted as the presence of nanometer sized particles, while characteristics in the visible range indicate micrometer sized particles. A possible conclusion is that dust particles are made of nanometric grains aggregated in large clusters (Mathis and Whiffen, 1989). Similar inference arose for other cosmic dust particles (Weidenschilling and Cuzzi, 1993). The inverse scattering problem-i.e. obtaining the complete description of a disordered object from its optical response-, cannot be handled for such complicated shapes. We can remember the dragon-tracks image given in a popular book (Bohren and Huffman, 2004, p. 10), and the question: can you "describe a dragon from its tracks"? Presently, the only practicable road is the direct way, that is to compare experimental data to calculations from tentative structures. However, it requires often a number of trial and error, is computationally demanding, and uniqueness of the solution is questionable.

Instead of trying to guess the complete particle structure in a single step, one could look for wavelength domains and scattering features providing partial, though definite, information about particle shape and material. The simplest example of such an approach is Rayleigh scattering: when the wavelength is much larger than the particle diameter, the scattering signal does not carry information about the inner structure of the particles, but about effective radius and average refractive index. Though incomplete, the information is valuable. The present work develops a similar idea,

Copyright (c) The Society of Geomagnetism and Earth, Planetary and Space Sciences (SGEPSS); The Seismological Society of Japan; The Volcanological Society of Japan; The Geodetic Society of Japan; The Japanese Society for Planetary Sciences; TERRAPUB.

doi:10.5047/eps.2013.03.011 beyond the Rayleigh approximation.

We consider below a composite particle made of two different compounds, and we ask: 1) what is the volume fraction of each compound? 2) which formation process is plausible? In this context, the coated sphere approximation is appropriate as it gives access to the composition of the 2compounds particle. Comparing the coated sphere theory to two generic disordered core-shell models, we determine wavelength domains in which specific information can be extracted. That way, we reinstate the Mie theory as a fundamental tool to obtain relevant information from particle scattering data, in a controlled manner. Other simple theories (e.g. the effective medium theory) or more advanced models (as presented in the Conclusion) could be considered as well.

\section{General Introduction to the Method}

The starting point is the suggestion by Mathis (Mathis, 1996) to consider the ratios between the optical cross sections of randomly oriented oblate spheroids of given shape and the corresponding cross sections of the sphere of equal volume. It appears that these ratios can be estimated simply for wavelengths much smaller or much larger than the size of the particle. Moreover, the ratios are essentially independent on the wavelength in the case of randomly oriented homogeneous ellipsoids with shape distribution (Min et al, 2003). Then, these optical ratios show relatively simple behaviors, and can be useful though restricted to simple geometrical shapes.

Generalizing this approach, we consider the optical efficiency factor, $Q$, of an ensemble of scattering particles. $Q$ is the ratio between the corresponding optical cross section and the area $\pi a_{\text {eff }}^{2}$, with $a_{\text {eff }}$ the solid-volume-equivalent radius (Shen et al, 2008). The actual value of $Q$ depends on the wavelength $\lambda$ and on the spatial distribution of the re- 

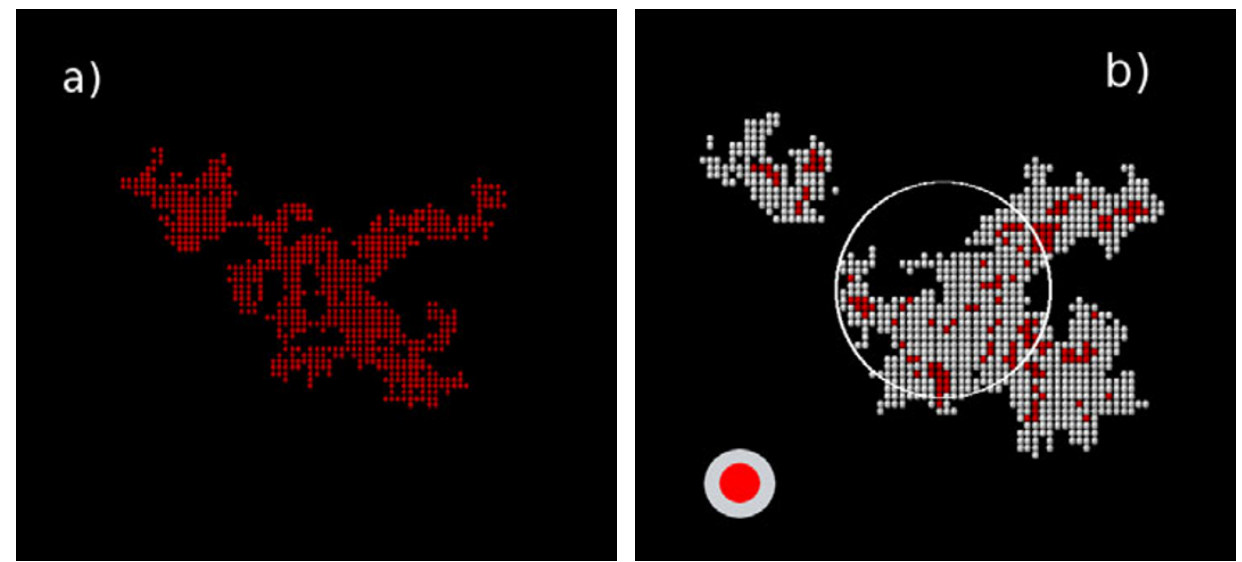

Fig. 1. $B C_{\mathrm{A}} B P_{\mathrm{B}}$ particle. a) plane projection of a fractal $B C_{\mathrm{A}}$ cluster of 2048 A-grains (red); b) cut of the $B C_{\mathrm{A}} B P_{\mathrm{B}}$ cluster made from the A-cluster randomly coated with $8192 \mathrm{~B}$-grains (grey). The white circle marks the effective radius of the particle (here much smaller than the cluster span). For comparison, cut of the coated sphere made of $f_{c}=20 \% \mathrm{~A}$ (red core) coated with $80 \% \mathrm{~B}$ (grey mantle), is shown at a smaller scale in the bottom left of the figure. Color online.

fractive index. Let $Q^{\text {approx }}(\lambda)$ be the value of $Q$ for a model approximating the particle structure. We introduce the ratio, $S(\lambda)$, as:

$$
Q(\lambda)=S(\lambda) \times Q^{\text {approx }}(\lambda)
$$

Then, we will calculate values of $Q(\lambda)$ for models of particles possibly representing real scatterers, and look for domains of the parameters where the behavior of $S(\lambda)$ is simple (the approximate theory will then be usable with a mere correction factor) or characteristic (possible and unambiguous interpretation of the features).

\section{The Method for Composite Particles}

Let us take the example of composite particle, that is assembly of homogeneous pieces of various chemical compositions (Henning, 1997). It might be a particle made of ice grains (Cuppen and Herbst, 2007), and of carbonaceous grains such as tholins (Sagan and Khare, 1979) or PAH (Ravindra et al, 2008), with either carbonaceous core (Wickramasinghe, 1965) or silicate core (Greenberg, 1989). However, we shall consider below generic materials instead of specific substances, since many features are expected to be almost insensitive to the chemical composition (Wright, 1987).

Then, we consider generically disordered particles made of grains of either compound $\mathrm{A}$, or compound $\mathrm{B}$, with an excess of $\mathrm{B}$ grains. The quality and optical parameters are as follows:

- constituent A is a sticky material, optically absorbing

- constituent B is a transparent volatile material with small optical absorption.

\subsection{Composition}

The present article is devoted to explaining a method, not to fitting current data, then the precise values of the parameters are not so important and we consider here typical values in order to keep the analysis simple. Following this idea, the volume fractions will be: $20 \%$ of $\mathrm{A}$ and $80 \%$ of $\mathrm{B}$, and the respective refractive indices:

$$
m_{\mathrm{A}}=1.9+0.5 i ; m_{\mathrm{B}}=1.3+10^{-3} i
$$

Note that we consider $m_{\mathrm{A}}$ and $m_{\mathrm{B}}$ independent of the wavelength. That way, any special feature depending on the wavelength will be a shape effect. In the following, $f_{c}$ denotes the volume fraction of the core.

\subsection{Generic shapes}

Clusters made of two kinds of domains result generally from a 2 -steps process (Helling and Woitke, 2006). According to the fractal theory (Jullien and Botet, 1987), formation of aggregated particles in rarefied medium may result from four different processes when melting can be neglected (Lin et al, 1987). The names given below refer to the formation modes, according to the combination/core/mantle/, and the coding (Meakin and Jullien, 1988): ' $B$ ' $\rightarrow$ 'Ballistic', ' $R$ ' $\rightarrow$ 'Reaction-limited', ' $C$ ' $\rightarrow$ Cluster-Cluster model, ' $P$ ' $\rightarrow$ Particle-Cluster model.

We focus on the two processes most likely to occur for sticky $\mathrm{A}$ and volatile $\mathrm{B}$, namely: $B C_{\mathrm{A}} B P_{\mathrm{B}}$ and $R P_{\mathrm{B}} B P_{\mathrm{A}}$, depending on whether $\mathrm{A}$ or $\mathrm{B}$ grains aggregate first.

- $B C_{\mathrm{A}} B P_{\mathrm{B}}$ process:

a cloud of sticky A-grains condensates in aggregates (Gelencsér, 2004). Since the clusters move freely, the Ballistic Cluster-Cluster Aggregation process (BaCCA) generates fractal particles, with the fractal dimension $D_{f} \simeq 1.9$ (Meakin and Jullien, 1988), see Fig. 1(a). In a second step, the A-particles cross a cloud made of dispersed B-grains, which come and stick on the particle surfaces (Ravey, 1975), producing B-coating through the Ballistic Particle-Cluster process (BaPCA) (Meakin, 1985). A final particle is shown on Fig. 1(b). It is made of a disordered fractal core of A, coated with B-grains.

- $R P_{\mathrm{B}} B P_{\mathrm{A}}$ process:

B-grains condensate first in clusters through the Reaction-limited Particle-Cluster Aggregation process (RPCA) since bonds between adjoining B-grains are weak (Jullien and Botet, 1987). These clusters are compact with rough surface, see Fig. 2(a). Then the Bclusters cross a cloud of sticky A-grains which go and form random coating following the Ballistic ParticleCluster process (BaPCA), see Fig. 2(b). Final particles 

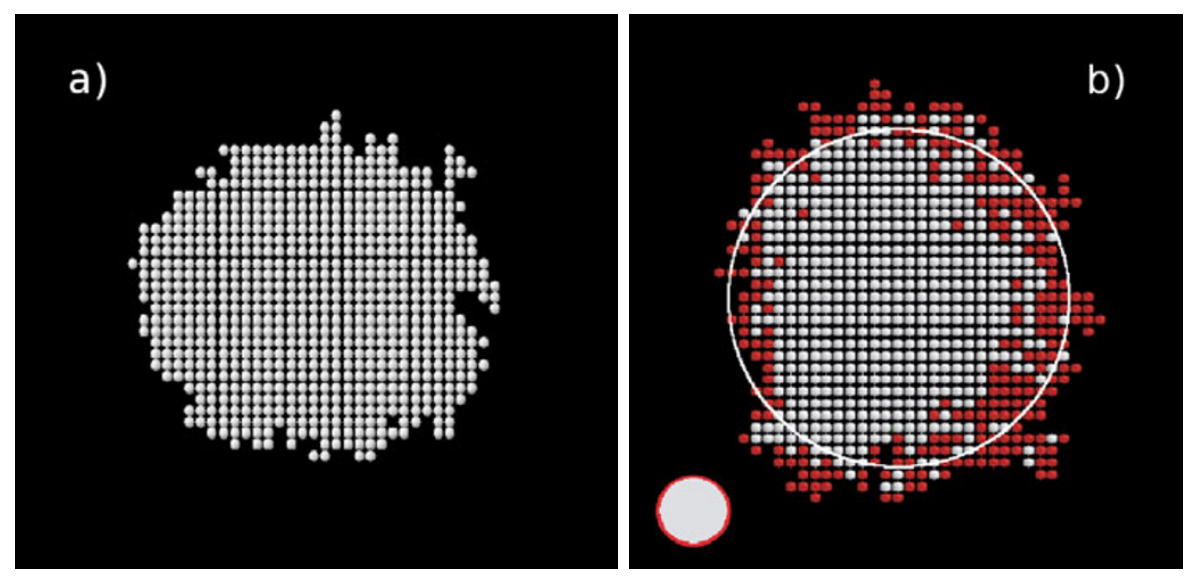

Fig. 2. $R P_{\mathrm{B}} B P_{\mathrm{A}}$ particle. a) plane projection of a disordered compact $R P_{\mathrm{B}}$ cluster of $8192 \mathrm{~B}$-grains (grey); b) cut of the $R P_{\mathrm{B}} B P_{\mathrm{A}}$ cluster made from the B-cluster randomly coated with 2048 A-grains (red). The white circle marks the cluster effective radius (here comparable to the overall cluster radius). For comparison, cut of the coated sphere made of $f_{c}=80 \% \mathrm{~B}$ (grey core) coated with $20 \%$ A (red mantle), is shown at a smaller scale in the bottom left of the figure. Color online.

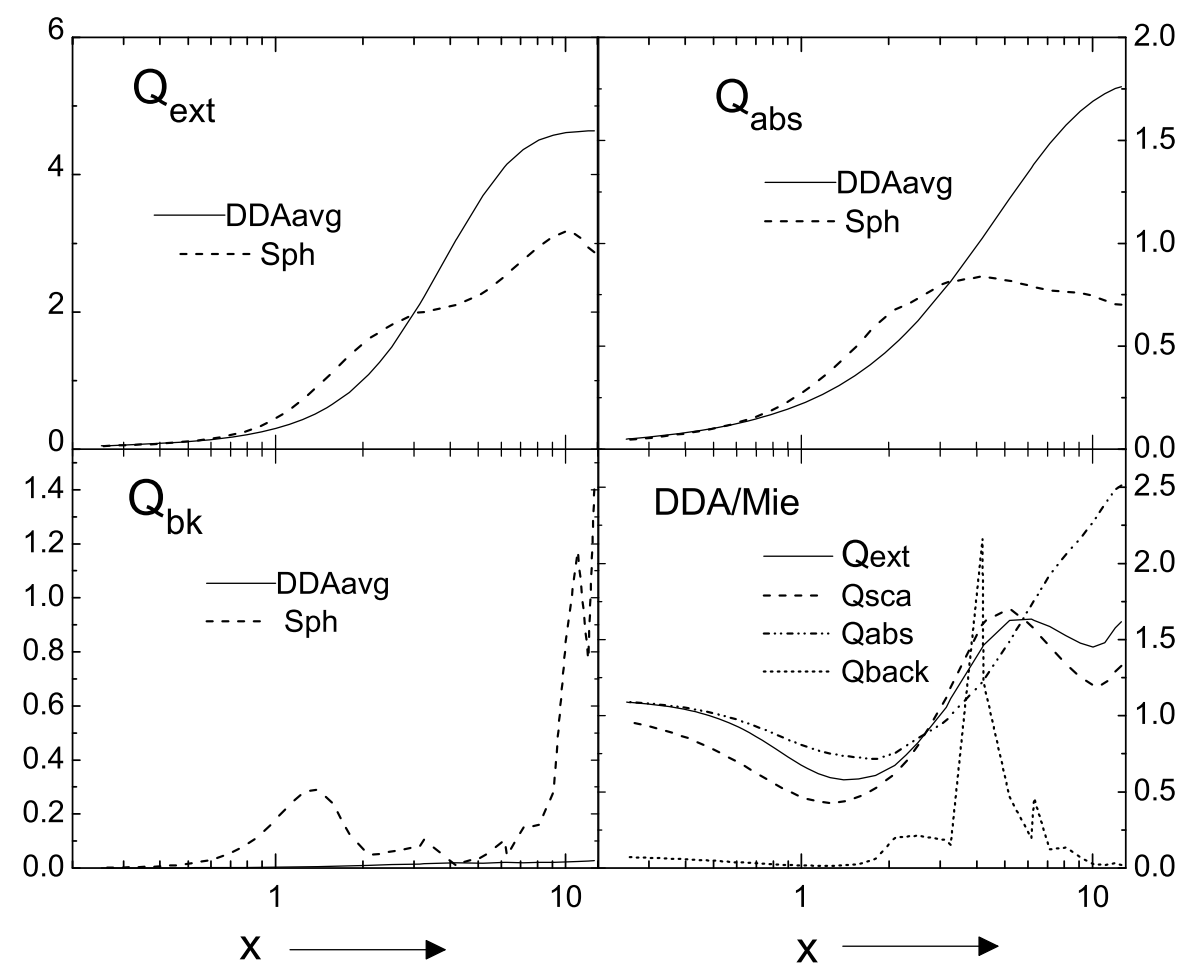

Fig. 3. Extinction (top-left), absorption (top-right), backscattering (bottom-left) averaged efficiencies for $25 B C_{\mathrm{A}} B P_{\mathrm{B}}$ clusters, $a_{\mathrm{eff}}=200 \mathrm{~nm}$ (full lines, 'DDAavg'), compared to the corresponding optical efficiencies of the coated sphere of same radius $a_{\mathrm{eff}}$, and composition (dashed lines, 'Sph'). The respective ratios $S(\lambda)$, as defined in (1), are on the bottom-right figure. The logarithmic scale for the optical parameter $x=2 \pi a_{\text {eff }} / \lambda$, is used to magnify the large- $\lambda$ range.

consist in a rough compact core of $\mathrm{B}$, coated with a thin shell of A.

\section{Optical Efficiency Factors for the Composite Particles}

Ensembles of particles were prepared according to each formation process. The particles all consist of $N=10240$ dipoles and effective radius $a_{\text {eff }}=200 \mathrm{~nm}$. Ensembleand angle-averaged optical efficiencies were computed over the wavelength range: $0.1 \mu \mathrm{m}<\lambda<5 \mu \mathrm{m}$, using the DDSCAT code v.7.2 (Draine and Flatau, 2012). The results depend on the non-dimensional size parameter $x=$
$2 \pi a_{\text {eff }} / \lambda$, and on the particle formation process.

4.1 Optical efficiency factors of disordered fractal $A$ cluster coated with $\mathrm{B}$-grains $\left(B C_{\mathrm{A}} \boldsymbol{B} \boldsymbol{P}_{\mathrm{B}}\right.$ process)

We first consider optical properties of fractal A-core with B-coating, as in Fig. 1. The computed extinction, absorption and backscattering efficiencies are compared with the values obtained with the BHCOAT code (Bohren and Huffman, 2004) for a spherical particle made of $\mathrm{A}\left(f_{c}=20 \%\right)$, coated with B $(80 \%)$.

- on Fig. 3, we see that both models (coated fractal and coated sphere) exhibit strong absorption in the UV domain. This is not due to feature in the refractive indices, as they do 


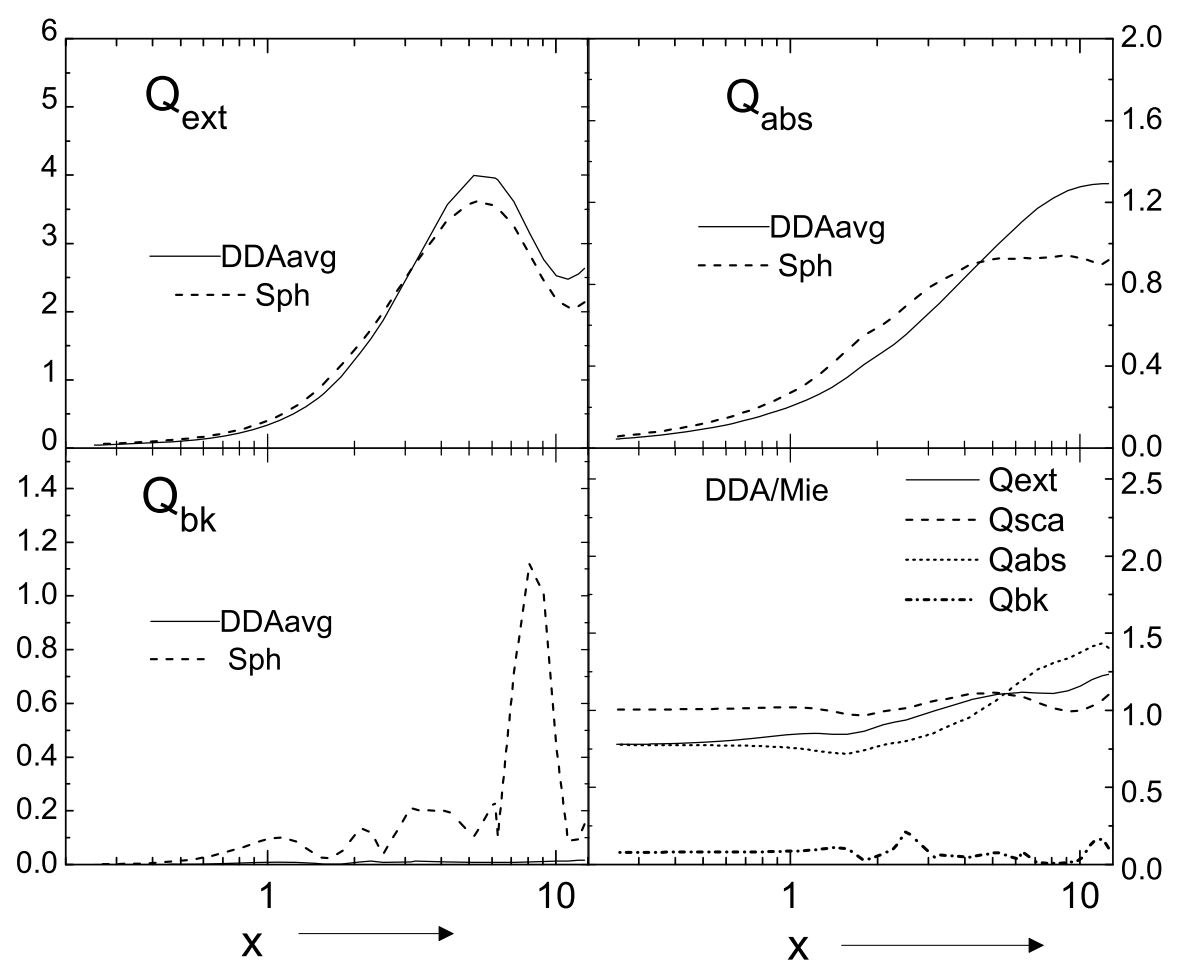

Fig. 4. Same legend as Fig. 3. Clusters built with the $R P_{\mathrm{B}} B P_{\mathrm{A}}$ process.

not depend, here, on the wavelength. Moreover, extinction and absorption efficiency factors of the fractal particles are about twice as large as for the coated sphere of same volume and composition. This is consistent with the absorption enhancement for the 'closed-cell' fractal aggregates (i.e. clusters of coated grains) compared with the 'open-cell' structures (core-shell structure) for large values of $x$ (Liou et al, 2011).

- the backscattering intensity is dramatically lowered for the coated fractal particles (Fig. 3 bottom-left) as the result of coherence loss of the backscattered waves, due to the structure irregularities. Such an effect is known in other domains of wave scattering (Botta et al, 2011).

4.2 Optical efficiency factors of rough compact $B$ cluster coated with A-grains $\left(\boldsymbol{R} \boldsymbol{P}_{\mathrm{B}} \boldsymbol{B} \boldsymbol{P}_{\mathrm{A}}\right.$ process)

The case of disordered compact particles (Fig. 2) is expected to resemble the coated sphere, apart from effects due to surface roughness. This can be checked on Fig. 4 where increase of a few $\%$ in the extinction and absorption efficiencies appear at wavelengths comparable to the roughness length-scale.

As previously, the backscattering intensity is strongly lowered at all wavelengths (Fig. 4 bottom-left).

\subsection{Discussion}

4.3.1 The large wavelength regime For wavelengths much larger than the effective particle radius (here, for: $x<$ $0.3)$, all the ratios $S(\lambda)$ are almost constant, independent of $x$ (Figs. 3 and 4). However, the values of the ratios depend on the model:

- the fractal absorbing cluster coated with transparent material, $\left(B C_{\mathrm{A}} B P_{\mathrm{B}}\right.$, Fig. 1), exhibits about the same absorbing cross section as the corresponding coated sphere, but a smaller scattering cross section, namely:
$S_{\text {abs }}=1.1 \pm 0.05, S_{\text {sca }}=0.8 \pm 0.05$

- on the contrary, the rough transparent cluster coated with absorbing material, $\left(R P_{\mathrm{B}} B P_{\mathrm{A}}\right.$, Fig. 2), presents scattering cross section comparable to the corresponding coated sphere of same composition, but the absorbing cross section is significantly smaller: $S_{\mathrm{abs}}=$ $0.77 \pm 0.05, S_{\text {sca }}=1.1 \pm 0.05$

These particular values of the ratios $S$ are independent on $\lambda$ but depend on $f_{c}$ and on the formation process. Let $S(\infty)$ be the limit ratios for a given formation process.

Suppose that, for a particle model, we had forgotten the values of the effective radius $a_{\text {eff }}$ and of the core volume fraction $f_{c}$, then we could determine them from the two equations in the small- $x$ range: $Q_{\text {abs }}^{\text {coated sphere }}=$ $Q_{\text {abs }} / S_{\text {abs }}(\infty), Q_{\text {sca }}^{\text {coated sphere }}=Q_{\text {sca }} / S_{\text {sca }}(\infty)$. In other words, once $S_{\mathrm{abs}, \mathrm{sca}}(\infty)$ known for a given model, analysis of real data requires only use of the observational data and of the Rayleigh formula for the coated sphere:

$$
\begin{gathered}
Q_{\mathrm{abs}}=4 x \operatorname{Im}\{\rho\} ; Q_{\mathrm{sca}}=\frac{8}{3} x^{4}|\rho|^{2} \\
\rho=\frac{\left(m_{B}^{2}-1\right)\left(m_{A}^{2}+2 m_{B}^{2}\right)+f_{c}\left(2 m_{B}^{2}-1\right)\left(m_{A}^{2}-m_{B}^{2}\right)}{\left(m_{B}^{2}+2\right)\left(m_{A}^{2}+2 m_{B}^{2}\right)+2 f_{c}\left(m_{B}^{2}-1\right)\left(m_{A}^{2}-m_{B}^{2}\right)}
\end{gathered}
$$

This gives direct information about the scatterers: the disordered particles are made of $4 \pi a_{\text {eff }}^{3} f_{c} / 3$ core volume, and $4 \pi a_{\text {eff }}^{3}\left(1-f_{c}\right) / 3$ coating volume.

4.3.2 The UV-shift Information about the particle formation process derives from the efficiency data in the range $1<x<6$. Indeed, we note the very different behaviors of the ratios $S(\lambda)$ for the two models (Fig. 3 and Fig. 4 bottom-right): the absorbing fractal core coated with transparent compound exhibits shift of the absorption and extinction efficiency factors peaks to the UV, compared to the 
coated sphere of same composition. It results in a characteristic dip-and-rise shape of the $S(\lambda)$ function in this range. Such a feature is totally absent for the $R P_{\mathrm{B}} B P_{\mathrm{A}}$ model.

4.3.3 Global information More generally, qualitative agreement between disordered composite particles and the coated sphere (such as in Fig. 4) is the sign of a compact core. Moreover, $S(\lambda)$ appears in this case to be a smooth correction factor ( $\neq 1$ in general) with meaningful values which can be used to determine the effective radius and the core volume of the scattering particles (large wavelengths) and give insights into the surface roughness (small wavelengths).

On the other hand, discrepancies between the coated sphere model and observational data carry information too. The case of the non-monotonic shape of the $S(\lambda)$ function-such as in Fig. 3-, signs UV shift of the scattering and absorbing peaks resulting from the presence of many small structures. Generally, deviations appear for the wavelengths of same order as the typical distance between sub-structures (Iati et al, 2004). These differences are naturally highlighted on $S(\lambda)$ features.

\section{Conclusion}

The motivation of this work was to show how a simple model (here: the coated sphere model) compare with the structure of complex dust particles. Agreement between observational data and the simple model in suitable parameter ranges, allows determination of the relevant parameters characterizing the model, while discrepancies are used to understand the geometry of the scatterers, as they correspond to shape effects (i.e. deviations from the shape considered in the simple model).

As illustration, we used models of 2-compounds composite particles of given composition (namely: absorbing A compound, transparent B compound, and excess of B grains) to exemplify which information are obtained from comparison to the coated sphere of same composition.

Other models depending on a few number of parameters could be used as well. A more sophisticated example is given in Kimura et al (2003), with aggregates of 1compound monodispersed grains. Indeed, the optical properties of these numerical particles were shown to be fairly independent of the cluster shape and size, for the grain radius and refractive index expected for cometary dust particles. Then, after characterizing wavelength ranges and optical quantities which are sensitive to one of the parameters and not the others, one should be able to build inversemethod tools for grain radius and refractive index determination from observational data.

Acknowledgments. We thank N. Voshchinnikov (referee) and H. Kimura (editor) for very many constructive comments and suggestions, making the text significantly improved. R. B. would like to thank E. Hadamcik and R. Gupta for helpful discussions during visit of IUCAA (Pune, India). We also thank an anonymous referee for his/her helpful remarks about a number of unclear points in the first version of the manuscript. This work was supported by France-India CEFIPRA project No 4407-A "Cometary grains: observations and simulations".

\section{References}

Bohren, C. E. and D. R. Huffman, Absorption and Scattering of Light by Small Particles, 544 pp, Wiley-VCH, Verlag, 2004.

Botta, G., K. Aydin, J. Verlinde, A. E. Avramov, A. S. Ackerman, A. M. Fridlind, G. M. McFarquhar, and M. Wolde, Millimeter wave scattering from ice crystal aggregates: Comparing cloud model simulations with $\mathrm{X}$ - and Ka-band radar measurements, J. Geophys. Res. 116, D00T04, $1-13,2011$.

Cuppen, H. M. and E. Herbst, Simulation of the Formation and Morphology of Ice Mantles on Interstellar Grains, Astrophys. J., 668, 294-309, 2007.

Draine, B. T. and P. J. Flatau, User Guide for the Discrete Dipole Approximation Code DDSCAT 7.2, http://arxiv.org/abs/1202.3424, 2012.

Gelencsér, A., Carbonaceous Aerosols, 362 pp, Atmospheric and Oceanographic Sciences Library, Springer, Dordrecht, 2004.

Greenberg, J. M., From interstellar dust to comet dust and inteplanetary particles, Highlights Astron., 8, 241-250, 1989.

Helling, Ch. and P. Woitke, Dust in brown dwarfs V. Growth and evaporation of dirty dust grains, Astron. Astrophys., 455, 325-338, 2006.

Henning, Th., Interstellar dust grains - an overview, in Molecules in Astrophysics: Probes and Processes, IAU Symp. No.178, 343-356, edited by E.F. van Dishoeck, Kluwer Acad. Publ., Dordrecht, 1997.

Iati, M. A., A. Giusto, R. Saija, F. Borghese, and P. Denti, Optical Properties of Composite Interstellar Grains: A Morphological Analysis, Astrophys. J., 615, 286-299, 2004.

Jullien, R. and R. Botet, Aggregation and Fractal Aggregates, 130 pp, World Scientific Publishing, Singapore, 1987.

Kimura, H., L. Kolokolova, and I. Mann, Optical properties of cometary dust-Constraints from numerical studies on light scattering by aggregate particles-, Astron. Astrophys., 407, L5-L8, 2003.

Lin, M. Y., H. M. Lindsay, D. A. Weitz, R. C. Ball, R. Klein, and P. Meakin, Universality of fractal aggregates as probed by light scattering, Proc. $R$. Soc. Lond. A, 423, 71-87, 1987.

Liou, K. N., Y. Takano, and P. Yang, Light absorption and scattering by aggregates: application to black carbon and snow grain, J. Quant. Spectrosc. Radiat. Transfer, 112, 1581-1594, 2011.

Mathis, J. S., Dust models with tight abundance constraints, Astrophys. J., 472, 643-655, 1996.

Mathis, J. S. and G. Whiffen, Composite interstellar grains, Astrophys. J., 341, 808-822, 1989.

Meakin, P., Accretion processes with linear particle trajectories, J. Coll. Interf. Sci., 105, 240-246, 1985.

Meakin, P. and R. Jullien, The effects of restructuring on the geometry of clusters formed by diffusion-limited, ballistic, and reaction-limited cluster-cluster aggregation, J. Chem. Phys., 89, 246-250, 1988.

Min, M., J. W. Hovenier, and A. de Koter, Shape effects in scattering and absorption by randomly oriented particles small compared to the wavelength, Astron. Astrophys., 404, 35-46, 2003.

Ravey, J. C., Computer simulation of morphological and optical properties of aggregates of spheres, J. Coll. Interf. Sci., 50, 545-558, 1975.

Ravindra, K., R. Sokhi, and R. van Grieken, Atmospheric polycyclic aromatic hydrocarbons: Source attribution, emission factors and regulation, Atmos. Environ., 42, 2895-2921, 2008.

Sagan, C. and B. N. Khare, Tholins: organic chemistry of interstellar grains and gas, Nature, 277, 102-107, 1979.

Shen, Y., B. T. Draine, and E. T. Johnson, Modeling porous dust grains with ballistic aggregates I. Geometry and optical properties, Astrophys. J., 689, 260-275, 2008.

Weidenschilling, S. J. and J. N. Cuzzi, Formation of planetesimals in the solar nebula, in Protostars and Planets III, edited by E. H. Levy and J. I. Lunine, 1031-1060, Arizona Press, Tucson, 1993.

Wickramasinghe, N. C., On the growth and destruction of ice mantles on interstellar graphite grains, Mon. Nat. R. Astron. Soc., 131, 177-190, 1965.

Wright, E. L., Long-wavelength absorption by fractal dust grains, Astrophys. J., 320, 818-824, 1987.

R. S. Botet (e-mail: robert.botet@u-psud.fr) and R. K. Rai 